\title{
Optical Sensitivity Gain in Silica-Coated Plasmonic Nanostructures
}

\author{
Francesco Floris, ${ }^{\dagger}$ Cristiana Figus, ${ }^{\dagger}$ Lucia Fornasari, ${ }^{\dagger}$ Maddalena Patrini, ${ }^{\dagger}$ Paola Pellacani, ${ }^{\S}$ \\ Gerardo Marchesini, ${ }^{\S}$ Andrea Valsesia, ${ }^{\S}$ Flavia Artizzu, ${ }^{\ddagger}, \|$ Daniela Marongiu, ${ }^{\ddagger}$ Michele Saba, ${ }^{\ddagger}$ \\ Andrea Mura, ${ }^{\ddagger}$ Giovanni Bongiovanni, ${ }^{\ddagger}$ Franco Marabelli, ${ }^{\dagger}$ and Francesco Quochi* ${ }^{\ddagger}$ \\ ${ }^{\dagger}$ Dipartimento di Fisica, Università degli Studi di Pavia, Via Bassi 6, I-27100 Pavia, Italy \\ ${ }^{\ddagger}$ Dipartimento di Fisica, Università degli Studi di Cagliari, S.P. Monserrato-Sestu Km 0,700, I-09042 Monserrato, Cagliari, Italy \\ ${ }^{\S}$ Plasmore SRL, Via Deledda 4, I-21020 Ranco, Varese, Italy \\ "Dipartimento di Scienze Chimiche e Geologiche, Università degli Studi di Cagliari, S.P. Monserrato-Sestu Km 0,700, I-09042 \\ Monserrato, Cagliari, Italy
}

\section{Supporting Information}

ABSTRACT: Ultrathin films of silica realized by sol-gel synthesis and dip-coating techniques were successfully applied to predefined metal/polymer plasmonic nanostructures to spectrally tune their resonance modes and to increase their sensitivity to local refractive index changes. Plasmon resonance spectral shifts up to $100 \mathrm{~nm}$ with slope efficiencies of $\sim 8 \mathrm{~nm} / \mathrm{nm}$ for increasing layer thickness were attained. In the ultrathin layer regime $(<10 \mathrm{~nm})$, which could be reached by suitable dilution of the silica precursors and optimization of the deposition speed, the sensitivity of the main plasmonic resonance to refractive index changes in aqueous solution could be increased by over $50 \%$ with respect to the bare plasmonic chip. Numerical simulations supported experimental data and unveiled the mechanism responsible for the optical sensitivity gain, proving an effective tool in the design of high-performance plasmonic sensors.

SECTION: Plasmonics, Optical Materials, and Hard Matter
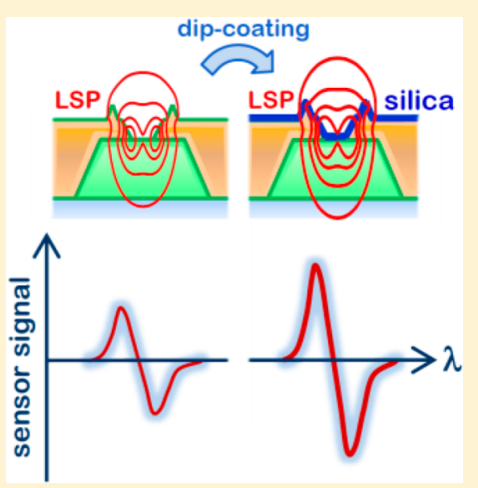

S urface plasmons are electromagnetic modes combined to coherent oscillations of free electrons in metals that have been deeply investigated for improving the performances of photonic and optoelectronic devices, including light-emitting diodes $^{1,2}$ and solar cells, ${ }^{3,4}$ and have enabled the realization of nanoscale laser sources. ${ }^{5,6}$ Local surface plasmons (LSPs) and surface plasmon polaritons (SPPs) are very sensitive to local refractive index variations in the surrounding medium. ${ }^{7,8}$ This sensitivity makes dielectric coating of plasmonic nanoparticles and nanostructures a convenient and powerful tool for harnessing surface plasmon resonances for specific applications in optoelectronics and optical sensing technologies. Overall, ultrathin dielectric coating (shell) layers with a thickness comparable to or smaller than the plasmon field penetration depth in the dielectric medium should bring several functionalities to the plasmonic system, that is, (i) spectral tuning of the plasmon resonances; (ii) encapsulation of the metal phase of the particle/structure and, hence, protection against external agents like corrosion; (iii) prevention of exciton quenching in the molecular/semiconducting material phase of the system near the metal interface. ${ }^{9}$

Several materials have been considered for realizing dielectric/insulating layers in plasmonic devices; among them, silica is one of the most widely utilized as it is relatively inert and insoluble, can be synthesized and processed into thin films by simple and versatile methods such as sol-gel synthesis and dip-coating, it can be heavily doped, ${ }^{10,11}$ and its surface is highly functionalizable. ${ }^{12,13}$ Silica-coated nanoparticles have, in fact, been widely used for, for example, enhancing light absorption in solar cells, ${ }^{14}$ controlling resonance energy transfer processes between encapsulated chromophores, ${ }^{15}$ as well as in plasmonic-magnetic nanodevices for bioapplications. ${ }^{16}$ Recently, the sol-gel method has been proven effective for the fabrication of thin silica layers on plasmonic platforms. Sol-gel silica coating layers on gold nanorod planar arrays have displayed chemical stability in many organic solvents and allowed for enhancing the optical emission quantum yield of chromophores immobilized at the device surface. ${ }^{17}$

Here, we report on a combined experimental/computational study of the optical performance of planar plasmonic nanostructures coated with ultrathin silica layers. We demonstrate sol-gel synthesis and dip-coating as versatile and highly controllable techniques for the deposition of morphology-preserving, ultrathin silica coating layers down to the 10 $\mathrm{nm}$ thickness range, where surface plasmon resonance shift displays maximum sensitivity to increasing coating thickness. By correlating experimental optical spectra to numerically calculated electromagnetic field distributions for the LSP

Received: July 11, 2014

Accepted: August 8, 2014

Published: August 8, 2014 
resonance, we unveil mechanisms of the plasmon-light interaction in the nanostructures and demonstrate how to use sol-gel silica layers to increase plasmon sensitivity to surface dielectric perturbations for advanced applications in chemical sensing and biosensing. A dielectric coating layer is usually necessary for device surface protection, but it causes degradation of the device performance by reducing the nearfield interaction between the plasmonic structure and the external environment. We show that in the ultrathin layer regime the dielectric coating, instead of being a hindrance, can increase the effectiveness of a plasmonic structure.

The investigated plasmonic nanostructures consist of a hexagonal lattice of poly(methyl methacrylate) (PMMA) pillars-about $500 \mathrm{~nm}$ in period- embedded in an optically thick gold film deposited on a glass substrate. Such nanostructures, schematically depicted on top of Figure 1,

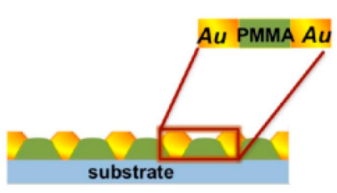

uncoated structure
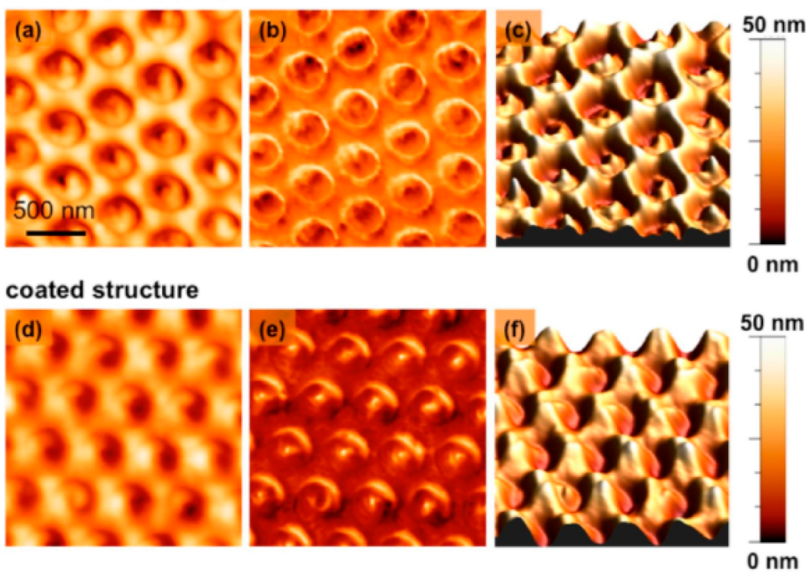

Figure 1. AFM imaging of a hybrid organic-inorganic plasmonic nanostructure consisting of an hexagonal lattice of polymeric (PMMA) pillars embedded in an optically thick $(80 \mathrm{~nm})$ gold film deposited on a glass substrate, before and after coating with an ultrathin $(\sim 6 \mathrm{~nm})$ layer of silica (blue layer in the uppermost right sketch). (a,d) Surface topography (height) images of the uncoated and coated nanostructure; $(\mathrm{b}, \mathrm{e})$ corresponding phase-contrast images; $(\mathrm{c}, \mathrm{f})$ three-dimensional views of surface topography images $(\mathrm{a}, \mathrm{d})$.

were fabricated and characterized for biosensing applications; ${ }^{18,19}$ the process of surface coating with a silica layer is also illustrated. Detailed morphological characterization was carried out by scanning electron microscopy (SEM). ${ }^{20}$ The combination of acid-catalyzed sol-gel synthesis using inorganic (tetraethoxysilane, TEOS) precursors and dip-coating allowed for depositing ultrathin silica layers of controlled thickness on the plasmonic nanostructures. A critical withdrawal speed of $\sim 50 \mathrm{~mm} / \mathrm{min}$, at which capillary regime and draining regime intersect each other, ${ }^{21}$ was chosen in the dip-coating procedure in order to minimize the silica layer thickness and thickness sensitivity to withdrawal speed variations/fluctuations. In addition, suitable volume dilution of the silica precursors' solution (Supporting Information, Figure S1) was adopted to tune the thickness of the films deposited at critical speed to values lower than $10 \mathrm{~nm}$. The silica coating thickness was measured on reference films deposited on soda-lime glass substrates. The thickness of the silica films resulted to be controllable at the nanometer scale with $1 \mathrm{~nm}$ absolute reproducibility.

The comparison between the morphology of an uncoated nanostructure and that of the same nanostructure after coating with an ultrathin $(\sim 6 \mathrm{~nm})$ layer of silica was performed by atomic-force microscopy (AFM); 2D/3D topographic and phase contrast images, shown in Figure 1, demonstrate that the coating was a spatially homogeneous process preserving the salient features of the morphology of the bare nanostructure, whereas some degree of smoothing of topographic details occurred; in particular, the tops of the polymeric pillars, visible in the topography of the uncoated nanostructure, were no longer observable after coating (panels $\mathrm{c}$ and $\mathrm{f}$ ).

It is well known that an increase in the real part of the permittivity of the dielectric medium at the metal/dielectric interface causes a redshift of the plasmon resonance to occur. ${ }^{22}$ As reported in Figure 2a, coating the periodic plasmonic nanostructure with a $\sim 6-\mathrm{nm}$-thick dielectric layer of silica results in a large $(\sim 40 \mathrm{~nm})$ redshift of the fundamental LSP
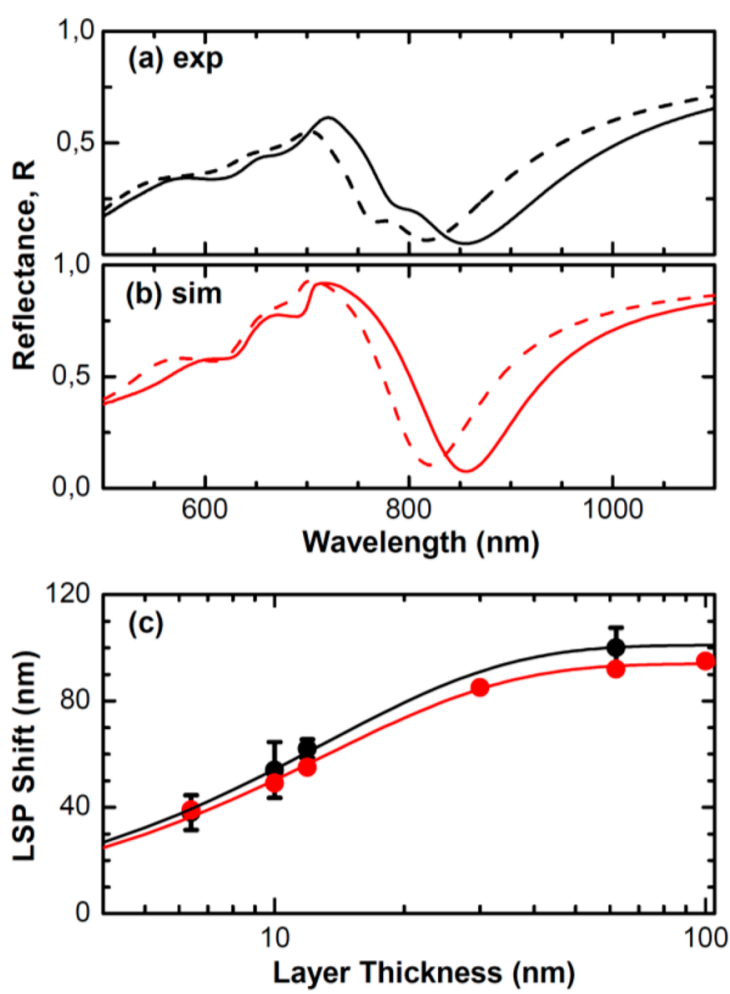

Figure 2. (a) Experimental reflectance spectrum of a plasmonic nanostructure before coating (dashed line) and upon deposition of a $\sim 6$-nm-thick silica layer (solid line). (b) FDTD simulation of the reflectance spectrum before and after coating. (c) Wavelength shift of the fundamental LSP resonance versus silica layer thickness. Black symbols are experimental values, averaged over several positions of the optical spot across the plasmonic chip surface; red symbols are the shift values inferred from FDTD simulations of the reflectance spectra. Continuous lines are best fits of the "charging" function $f(t)=A[1-$ $\left.\exp \left(-t / t_{\mathrm{c}}\right)\right]$ to the data, where $A$ is an amplitude and $t_{\mathrm{c}}$ is a characteristic thickness. Best fits yielded $A=101(2) \mathrm{nm}, t_{\mathrm{c}}=13.0(4)$ $\mathrm{nm}$ for the experimental data, and $A=94(1) \mathrm{nm}, t_{\mathrm{c}}=13.1(5) \mathrm{nm}$ for the simulated data, from which sensitivity of plasmon resonance shift to small thickness variations $\left(S \cong A / t_{c}\right)$ was inferred to be $7.7(3)$ and $7.2(3) \mathrm{nm} / \mathrm{nm}$, respectively. 
resonance located at $\lambda_{\mathrm{r}}=820 \mathrm{~nm}$ (wavelength of the dip in the reflectance spectrum). The complex structure of resonances extending across the $600-800 \mathrm{~nm}$ wavelength interval, which arise from hybridization of the LSP mode with the singleinterface (glass/gold) SPP mode, and whose detailed account was reported elsewhere, ${ }^{16}$ also undergoes an overall red shift in the presence of the top silica layer. The optical reflectance spectra of the plasmonic nanostructure confirm that ultrathin dielectric coatings can be proficiently used to tailor complex surface plasmon resonance modes, widely shifting their resonance wavelengths in a controllable way. Figure $2 \mathrm{~b}$ shows finite-difference time-domain (FDTD) simulations of the reflectance spectra obtained with a model nanostructure shape based on AFM topography and refined to match LSP resonance peak wavelength and line width in the uncoated plasmonic chip. Simulations were able to account for the presence of two hybrid surface plasmon resonances in the 600$700 \mathrm{~nm}$ range, whereas the hybrid mode appearing as a shoulder on the low-wavelength side of the LSP mode (at $\lambda$ $\sim 760 \mathrm{~nm}$ ) could not be reproduced. The effectiveness of FDTD calculations in simulating our quasi-2D plasmonic structure is further demonstrated in Figure 2c, where the silicacoating-induced LSP resonance shift is plotted versus coating layer thickness. Silica thickness values, as well as its refractive index $n$, were previously inferred from curve fitting of spectroscopic ellipsometry and normal-incidence transmittance data taken on reference soda-lime glass substrates coated using the same deposition parameters used for coating the plasmonic nanostructures (Supporting Information, Figures S1 and S2). Experimentally, the LSP resonance redshift increases linearly at low layer thicknesses with a slope efficiency of $\sim 8 \mathrm{~nm} / \mathrm{nm}$ and saturates to a value $\sim 100 \mathrm{~nm}$ for thickness values $(\gtrsim 50 \mathrm{~nm})$ comparable to the penetration depth of the plasmon field intensity in silica (Figure 2c). Model calculations followed the same growth and saturation behavior with only a slight underestimate of the shift values. ${ }^{23}$

Further insight into the properties of the plasmonic nanostructures and their potential for optical sensing applications was gained from the simulations by inspecting the spatial distribution the LSP field intensity realized in the nanostructure both before and after silica coating, assuming either air or water as the medium filling the half-space above the system. Planar cross sections of the electric-field intensity calculated at the LSP resonance wavelength in the region surrounding the polymeric pillar are depicted in Figure 3 . When the ambient above the nanostructure is air, dielectric unbalance is realized between the upper-space region and the lower-space region, occupied by the $\mathrm{SiO}_{2}$ substrate, and the plasmon field intensity exhibits some degree of pinning at the lower pillar rim (the one in contact with the substrate). Upon application of the ultrathin layer of silica $(n \sim 1.43)$ on top of the structure (yellow layer in panel b), the dielectric constant unbalance is partly removed, the field intensity is greatly enhanced at the inner top rim of the pillar and the plasmon field extension into the upper space around the pillar axis is also increased. From such body of evidence it was inferred that the ultrathin silica coating not only provides chemical stabilization of the nanostructure surface and device protection against external contamination but also increases plasmon sensitivity to refractive index changes induced by physical agents present in close proximity of the nanostructure or by chemical/biological analytes adsorbed at the silica surface.
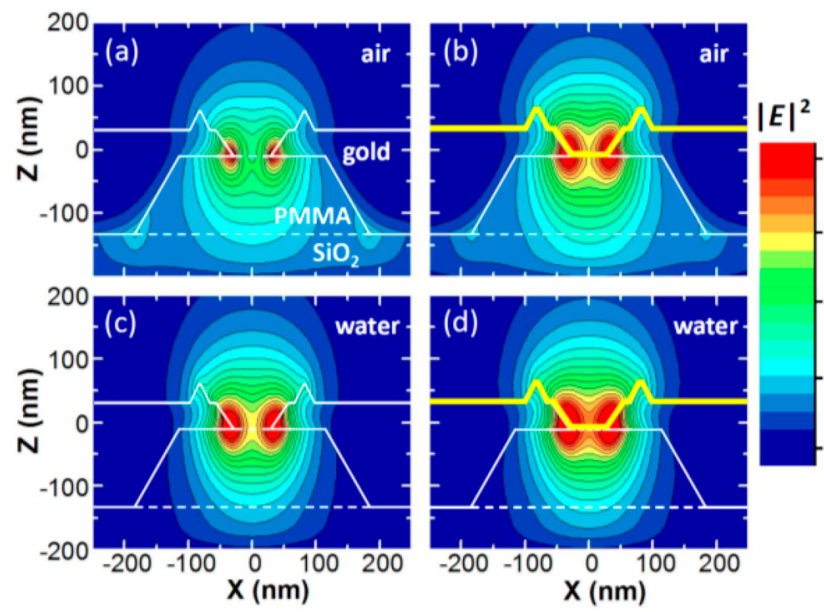

2

Figure 3. Spatial maps of the electric field intensity $|E|^{2}$, calculated at the fundamental LSP resonance wavelength $\left(\lambda_{\mathrm{r}}\right)$ corresponding to the main dip of the simulated reflectance spectrum. White lines highlight the contours between different materials of the nanostructure, whose lattice period is $500 \mathrm{~nm}$. Continuous lines highlight the metal/ dielectric interfaces, whereas dashed lines mark the separation between different dielectric materials. (a) Bare plasmonic nanostructure in air, $\lambda_{\mathrm{r}}=822 \mathrm{~nm}$; (b) coated nanostructure in air, $\lambda_{\mathrm{r}}=852 \mathrm{~nm}$. Thick yellow contour line depicts a $\sim 6$-nm-thick silica layer deposited on top of the nanostructure. (c,d) Same as $(a, b)$ but in water $\left(\lambda_{\mathrm{r}}=874\right.$ and $894 \mathrm{~nm}$, respectively). The E-field intensity is in arbitrary units.

The change in electromagnetic field intensity distribution induced by the ultrathin silica coating layer was qualitatively reproduced when the upper space was filled with water (panels $c$ and $d$ ), which is particularly interesting for optical sensing applications. Water $(n \sim 1.33)$ somewhat reduces the aforementioned dielectric unbalance and causes a larger portion of the plasmon mode to concentrate in the region above the top pillar aperture, as well as a small decrease in sensitivity of the plasmon field distribution to the presence of the silica coating layer.

The potential of sol-gel silica coatings for optical sensing was assessed through direct measurement of the sensitivity of plasmonic nanostructures to refractive index changes in aqueous solution; tests were conducted on the same nanostructure chips, before and after coating, in a flow cell for varying concentration of organic molecules and ionic species. Figure 4 shows results obtained when ethanol acts as the refractive index perturber in ultrapure (Milli-Q grade) water. The response of the system was quantified as the normalized reflectance spectrum, $R / R_{0}$, where $R(\lambda)$ is the perturbed spectrum (measured in aqueous solution of ethanol) and $R_{0}(\lambda)$ the unperturbed spectrum measured in ultrapure water. A concentration of ethanol in solution resulted in a spectral shift of the LSP resonance, which appeared in the normalized reflectance spectrum as a dispersive signal centered at the initial (unperturbed) resonance wavelength $\lambda_{\mathrm{r}}$; the peakto-valley amplitude difference of the dispersive signal could be used as the ethanol sensor signal. ${ }^{21}$ Figure $4 \mathrm{a}$ shows the experimental and simulated $R / R_{0}$ spectra at given ethanol concentration $(c \sim 1.1 \mathrm{M})$. The presence of many resonances related to hybridized plasmon modes yielded a complex structure of dispersive signals extending down to $550 \mathrm{~nm}$; our attention focused on the fundamental sigmoidal-like line shape arising from the LSP resonance, centered at $\lambda \sim 860 \mathrm{~nm}$. FDTD simulations were able to reproduce the main features of 


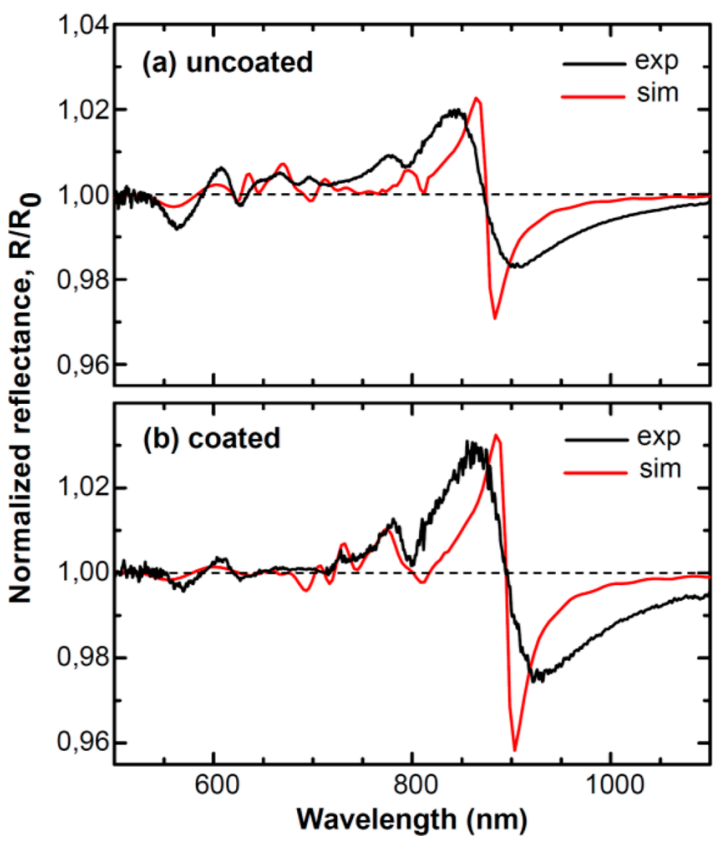

Figure 4. Normalized reflectance spectra $\left(R / R_{0}\right)$ of a plasmonic nanostructure chip obtained in optical sensing test. $R_{0}$ is the reference reflectance spectrum measured with the chip in ultrapure water. $R$ is the spectrum corresponding to a refractive index variation $\Delta n=3 \times$ $10^{-3}$ RIU produced by a concentration $c \sim 1.1 \mathrm{M}$ of ethanol in water. Panel (a) refers to the uncoated nanostructure, whereas panel (b) refers to the nanostructured chip coated with a $\sim 6$-nm-thick silica layer. Black lines: experiment; red lines: FDTD simulations.

the experimental spectra; the dispersive signal amplitudes were slightly overestimated as a possible consequence of the occurrence of disorder-induced light scattering in the real nanostructures (not accounted for in the numerical calculations), which systematically decreases the reflectance value at all wavelengths, broadening the signal line shape and attenuating its amplitude.

In line with numerical findings based on the FDTD plasmonfield simulations shown in Figure 3, the presence of an ultrathin silica coating layer enhances plasmon sensitivity to variations in refractive index of water (of the order of $10^{-3}$ RIU), as demonstrated by the larger signal amplitude exhibited by a given nanostructure chip after the coating process (Figure $4 \mathrm{~b}$ ). A thorough characterization of the plasmon chip response to changes in water refractive index yielded $\sim 56 \%$ gain in slope efficiency upon deposition of a $\sim 6$-nm-thick silica layer (Supporting Information, Figure S3). Results obtained on the same sensor chip with other solute molecules (glycerol and $\mathrm{NaCl}$ ) confirmed sensitivity enhancements over $20 \%$ in both cases.

We reported optical sensitivity gain, both experimentally and theoretically, in detecting small concentrations of organic molecules in water, however the approach used could be easily extended to the sensing of biological molecules. The silica surface can be functionalized with organosilanes having chemical affinity to biological molecules of interest; the thickness of the adsorbing functional layer would determine the effective change in refractive index induced by adsorbed molecules, ${ }^{24}$ while the underlying silica layer leading to enhancement of the plasmonic response to such refractive index change.
In summary, our experimental findings and numerical simulations demonstrated the potential of ultrathin coating layers on predefined plasmonic platforms for optical sensing applications. The simple and cost-effective techniques of sol-gel synthesis and dip coating were successfully applied to the deposition of well-controlled and reproducible silica layers less than $10 \mathrm{~nm}$ in thickness, much thinner than plasmon-fieldintensity penetration depth above the surface. This turned out to be a powerful method to encapsulate the plasmonic chip and tune predefined plasmonic resonances to the most convenient wavelengths for operating an optical sensor. While providing ease of plasmon-resonance tunability by tens of nanometers, the ultrathin coating layer enhances the plasmon sensitivity to refractive index variations by tens of percents. Experiments are currently in progress at our laboratories on plasmonicnanostructure biosensors with functionalized silica surfaces.

\section{EXPERIMENTAL AND COMPUTATIONAL METHODS}

Fabrication of plasmonic nanostructures. Plasmonic nanostructure chips were realized through colloidal lithography on glass substrates and consist of 2D arrays of PMMA truncated conical pillars (height of $\sim 150 \mathrm{~nm}$ and diameter of $\sim 350 \mathrm{~nm}$ ) embedded into an optically thick $(\sim 80 \mathrm{~nm})$ gold layer. Fabrication details can be found in previous work. ${ }^{18,19}$

Silica synthesis and deposition. All reagents and solvents were purchased from Sigma-Aldrich. The sol was prepared by mixing tetraethoxysilane (TEOS, purity >99\%), ethanol absolute $(\mathrm{EtOH})$, and distilled water $\left(\mathrm{H}_{2} \mathrm{O}\right)$, under stirring in a molar ratio of $1: 9.8: 5.6$ at room temperature (RT) for $24 \mathrm{~h}$. Subsequently, $3 \mathrm{~mL}$ of acetonitrile $(\mathrm{MeCN})$ and $3 \mathrm{~mL}$ of EtOH were added to $9 \mathrm{~mL}$ of the sol, $\mathrm{HCl}$ was added until $\mathrm{pH}$ $\sim 4$ was reached, and the mixture was stirred at $50{ }^{\circ} \mathrm{C}$ for $1 \mathrm{~h}$. The sol was then transferred into a glass vial and cooled to RT. Diluted solutions were prepared by adding EtOH to the sol. Soda-lime glasses were employed as substrates to prepare reference silica films for thickness calibration. Before coating, the substrates were cleaned with water, soap, $\mathrm{H}_{2} \mathrm{O}$, acetone, and finally rinsed with isopropyl alcohol. Substrates were dipped in the sol for $25 \mathrm{~s}$ and the films were then deposited by dipcoating (ND-R rotatory coater, Nadetech Innovations) at withdrawal speeds ranging from 0.6 to $1000 \mathrm{~mm} / \mathrm{min}$. After deposition, films were dried at RT for $48 \mathrm{~h}$. The same procedure was used to deposit silica films onto the plasmonic nanostructure chips.

Morphological characterization. Surface topography of uncoated and coated plasmonic nanostructure chips was measured by AFM (Solver-Pro, NT-MDT). AFM measurements were performed using silicon tips in semicontact mode in air. Analysis of topographic and phase-contrast images was performed using WSxM 5.0 Develop 3.2 software. Thickness of reference silica films deposited on soda-lime glass substrates was also estimated by AFM topography across a film cut made with a sharp blade.

Optical characterization. Angle-resolved reflectance measurements as well as reflectance characterization of refractive index sensitivity in a flow cell on the bare and coated plasmonic samples were performed in the spectral range between 0.5 and $1.2 \mu \mathrm{m}$, using a homemade microreflectometer setup coupled to a Fourier-Transform spectrometer Bruker IFS66. Typical measurements required a few minutes to collect 256 scans with a resolution of $16 \mathrm{~cm}^{-1}$ with a standard silicon photodiode. Transmittance measurements were done using an Agilent Cary 
$6000 \mathrm{i}$ spectrophotometer at normal incidence in the $0.2-1.6$ $\mu \mathrm{m}$ wavelength range, and ellipsometry measurements at the angles of incidence of $55^{\circ}, 60^{\circ}$, and $65^{\circ}$ were carried out with a variable-angle spectroscopic ellipsometer (VASE, J. A. Woollam Co., Inc.) in the $0.25-2.5 \mu \mathrm{m}$ range (spot size of $200 \mu \mathrm{m}$ in diameter). The optical functions (refractive index, $n$, and extinction coefficient, $k$ ), as well as the thickness $(t)$ of silica layers deposited on soda-lime glass substrates, were obtained by simultaneous analysis of spectroscopic ellipsometry and transmittance spectra in the Vis-NIR region. All spectra were analyzed using WVASE32 software. A two-layer model for the samples (substrate coated with sol-gel silica film) and a generalized Cauchy-Urbach model for the optical functions were used to best fit the experimental data. The optical functions of the soda-lime glass were inferred from measurements taken on a bare substrate and subsequently used to fit $n$, $k$, and $t$ of the sol-gel silica films in the two-layer structures.

Numerical calculations. FDTD numerical simulations of the optical reflectance spectra and of the plasmon electric field distributions were performed using Lumerical FDTD Solutions software. The bare plasmonic nanostructure was modeled as a glass substrate overlaid with a gold film perforated by an array of PMMA truncated cones. All calculations were performed by using a unit cell comprising of five pillars of the nanostructure with a uniform mesh size of $1 \mathrm{~nm}$. An additional layer of silica was added on the surface top in the coated model. Optical functions of the top silica film were taken from the previous analysis of spectroscopic ellipsometry data, whereas PMMA and gold data were taken from literature and Lumerical database.

\section{ASSOCIATED CONTENT}

\section{S Supporting Information}

Dependence of sol-gel silica layer thickness on synthesis and deposition parameters, optical functions of sol-gel silica, sensitivity of uncoated and coated plasmonic nanostructure chips to refractive index changes are available. This material is available free of charge via the Internet at http://pubs.acs.org.

\section{AUTHOR INFORMATION}

\section{Corresponding Author}

*E-mail: quochi@unica.it.

\section{Notes}

The authors declare no competing financial interest.

\section{ACKNOWLEDGMENTS}

The Regione Autonoma della Sardegna is gratefully acknowledged for financial support through POR Sardegna FSE 20072013, L.R.7/2007 "Promozione della Ricerca Scientifica e dell'Innovazione Tecnologica in Sardegna” (Research Projects CRP3-114, CRP-17571, CRP-18353, CRP-18013, and CRP24978).

\section{REFERENCES}

(1) Kwon, M.-K.; Kim, J.-Y.; Kim, B.-H.; Park, I.-K.; Cho, C.-Y.; Chisu Byeon, C.; Park, S.-J. Surface-Plasmon-Enhanced Light-Emitting Diodes. Adv. Mater. 2008, 20, 1253-1257.

(2) Kim, J.-B.; Lee, J.-H.; Moon, C.-K.; Lim, S.-Y.; Kim, J.-J. Highly Enhanced Light Extraction from Surface Plasmonic Loss Minimized Organic Light-Emitting Diodes. Adv. Mater. 2013, 25, 3571-3577.

(3) Atwater, H. A.; Polman, A. Plasmonics for Improved Photovoltaic Devices. Nat. Mater. 2010, 9, 205-213.

(4) Green, M. A.; Pillai, S. Harnessing Plasmonics for Solar Cells. Nat. Photonics 2012, 6, 130-132.
(5) Ma, R.-M.; Oulton, R. F.; Sorger, V. J.; Zhang, X. Plasmon Lasers: Coherent Light Sources at Molecular Scales. Laser Photonics Rev. 2013, 7, $1-21$.

(6) Bravo-Abad, J.; Garcia-Vidal, F. J. Plasmonic Lasers: A Sense of Direction. Nat. Nanotechnology 2013, 8, 479-480.

(7) Mayer, K. M.; Hafner, J. H. Localized Surface Plasmon Resonance Sensors. Chem. Rev. 2011, 11, 3828-3857.

(8) Yu, Z.; Fan, S. Extraordinarily High Spectral Sensitivity in Refractive Index Sensors Using Multiple Optical Modes. Opt. Express 2011, 19, 10029-10040.

(9) Park, Y.; Pravitasari, A.; Raymond, J. E.; Batteas, J. D.; Son, D. H. Suppression of Quenching in Plasmon-Enhanced Luminescence via Rapid Intraparticle Energy Transfer in Doped Quantum Dots. ACS Nano 2013, 7, 10544-10551.

(10) Sanchez, C.; Lebeau, B.; Chaput, F.; Boilot, J.-P. Optical Properties of Functional Hybrid Organic-Inorganic Nanocomposites. Adv. Mater. 2003, 15, 1969-1994.

(11) Takahashi, M.; Figus, C.; Malfatti, L.; Tokuda, Y.; Yamamoto, K.; Yoko, T.; Kitanaga, T.; Tokudome, Y.; Innocenzi, P. Strain-Driven Self-Rolling of Hybrid Organic-Inorganic Microrolls: Interfaces with Self-Assembled Particles. NPG Asia Mater. 2012, 4, e22.

(12) Hoffmann, F.; Cornelius, M.; Morell, J.; Fröba, M. Silica-Based Mesoporous Organic-Inorganic Hybrid Materials. Angew. Chem., Int. Ed. 2006, 45, 3216-3251.

(13) Pinna, A.; Figus, C.; Lasio, B.; Piccinini, M.; Malfatti, L.; Innocenzi, P. Release of Ceria Nanoparticles Grafted on Hybrid Organic-Inorganic Films for Biomedical Application. ACS Appl. Mater. Interfaces 2012, 4, 3916-3922.

(14) Choi, H.; Lee, J.-P.; Ko, S.-J.; Jung, J.-W.; Park, H.; Yoo, S.; Park, O.; Jeong, J.-R.; Park, S.; Kim, J. Y. Multipositional Silica-Coated Nanoparticles for High-Performance Polymer Solar Cells. Nano Lett. 2013, 13, 2204-2208.

(15) Zhao, L.; Ming, T.; Shao, L.; Chen, H.; Wang, J. PlasmonControlled Förster Resonance Energy Transfer. J. Phys. Chem. C 2012, 116, 8287-8296.

(16) Sotiriou, G.; Hirt, A. M.; Lozach, P.-Y.; Teleki, A.; Krumeich, F.; Pratsinis, S. E. Hybrid, Silica-Coated, Janus-Like Plasmonic-Magnetic Nanoparticles. Chem. Mater. 2011, 23, 1985-1992.

(17) Yasukuni, R.; Ouhenia-Ouadahi, K.; Boubekeur-Lecaque, L.; Félidj, N.; Maurel, F.; Métivier, R.; Nakatani, K.; Aubard, J.; Grand, J. Silica-Coated Gold Nanorod Arrays for Nanoplasmonic Devices. Langmuir 2013, 29, 12633-12637.

(18) Giudicatti, S.; Marabelli, F.; Valsesia, A.; Pellacani, P.; Colpo, P.; Rossi, F. Interaction among Plasmonic Resonances in a Gold Film Embedding a Two-Dimensional Array of Polymeric Nanopillars. J. Opt. Soc. Am. B 2012, 29, 1641-1647.

(19) Bottazzi, B.; Fornasari, L.; Frangolho, A.; Giudicatti, S.; Mantovani, A.; Marabelli, F.; Marchesini, G.; Pellacani, P.; Therisod, R.; Valsesia, A. Multiplexed Label-Free Optical Biosensor for Medical Diagnostics. J. Biomed. Opt. 2014, 19, 017006.

(20) Giudicatti, S.; Marabelli, F.; Pellacani, P. Field Enhancement by Shaping Nanocavities in a Gold Film. Plasmonics 2013, 8, 975-981.

(21) Faustini, M.; Louis, B.; Albouy, P. A.; Kuemmel, M.; Grosso, D. Preparation of Sol-Gel Films by Dip-Coating in Extreme Conditions. J. Phys. Chem. C 2010, 114, 7637-7645.

(22) Chen, H.; Kou, X.; Yang, Z.; Ni, W.; Wang, J. Shape- and SizeDependent Refractive Index Sensitivity of Gold Nanoparticles. Langmuir 2008, 24, 5233-5237.

(23) On the basis of the collected AFM topographic images of the plasmonic structure, a different surface interaction of the sol-gel silica matrix was hypothesized with gold and PMMA and supposed to result in a larger silica thickness on the PMMA pillar tops. Allowing for a slightly inhomogeneous silica thickness in the FDTD calculations (5 $\mathrm{nm}$ on gold and $7 \mathrm{~nm}$ on PMMA for a thickness of $6 \mathrm{~nm}$ measured on the reference film deposited on glass substrate) actually led to a more refined agreement between experiment and simulation, and finely reproduced the measured LSP resonance spectral shift in the ultrathin silica layer regime, for thickness values smaller than $10 \mathrm{~nm}$. 
(24) Homola, J. Surface Plasmon Resonance Sensors for Detection of Chemical and Biological Species. Chem. Rev. 2008, 108, 462-493. 